\title{
A Case of Prostate Cancer Recurrence in the Bladder Wall after Radical Prostatectomy: The Role of Diffusion-Weighted and Dynamic Contrast-Enhanced Magnetic Resonance Imaging
}

\author{
Caterina Gaudiano ${ }^{1}$, Fiorenza Busato ${ }^{2}$, Carlo Cecchelli ${ }^{3}$, Riccardo Schiavina ${ }^{4}$, \\ Eugenio Brunocilla ${ }^{5}$, Giuseppe Martorana ${ }^{6}$, and Rita Golfieri ${ }^{7}$ \\ ${ }^{1,2,3,7}$ Radiology Unit, Department of Digestive Diseases and Internal Medicine; Sant'Orsola-Malpighi \\ Hospital, University of Bologna, Bologna, Italy \\ ${ }^{4,5,6}$ Department of Urology, University of Bologna, Sant'Orsola-Malpighi Hospital, Bologna, Italy
}

Correspondence should be addressed to: Caterina Gaudiano; caterina.gaudiano@aosp.bo.it

Received date: 24 March 2014; Accepted date: 19 May 2014; Published date: 31 August 2016

Academic editor: Michael Quentin

Copyright (c) 2016. Caterina Gaudiano, Fiorenza Busato, Carlo Cecchelli, Riccardo Schiavina,Eugenio Brunocilla, Giuseppe Martorana, and Rita Golfieri. Distributed under Creative Commons CC-BY 4.0

\begin{abstract}
The authors report a case of prostate cancer recurrence in the bladder wall after radical prostatectomy studied by magnetic resonance imaging with diffusion weighted and dynamic contrast-enhanced sequences. The apparent diffusion coefficient (ADC) and the Time-Intensity (T-I) curves have been used to characterize tumor tissue.Multiparametric endorectal-MRI plays an important role in evaluating pelvic regions for the detection of local recurrence after radical prostatectomy and can improve therapeutic planning in the case of "salvage" therapy.
\end{abstract}

Keywords: Diffusion weighted imaging; Dynamic contrast-enhanced imaging; Multiparametric endorectal-MRI; Prostate cancer recurrence; Radical prostatectomy

\section{Introduction}

Prostate carcinoma (PCa) is one of the most common cancers in males. Clinical screening of PCa, mainly based on serum prostatespecific antigen (PSA), has increased the detection of early-stage PCa which is curable with a radical retropubic prostatectomy

Cite this Article as: Caterina Gaudiano, Fiorenza Busato, Carlo Cecchelli, Riccardo Schiavina,Eugenio Brunocilla, Giuseppe Martorana, and Rita Golfieri (2016)," A Case of Prostate Cancer Recurrence in the Bladder Wall after Radical Prostatectomy: The Role of Diffusion-Weighted and Dynamic Contrast-Enhanced Magnetic Resonance Imaging ", International Journal of Research in Urology, Vol. 2016 (2016), Article ID 652137, DOI: $10.5171 / 2016.652137$ 
(RRP) [1]. After a successful RRP, the PSA usually becomes undetectable $(<0.1 \mathrm{ng} / \mathrm{mL})$ within one month, and in the presence of any subsequent increase, recurrent disease should be suspected $[1,2]$.

An increase in PSA level, however, cannot provide precise information regarding the location of relapse, which can be local (in the prostatic bed) or systemic (skeletal or in other organs). These two conditions involve different therapies: local salvage therapy (surgery/radiotherapy) in the first case and systemic therapy (hormonotherapy) in the second case $[3,4]$.

The potential role of dynamic contrastenhanced (DCE) MRI in the detection of PCa recurrences after RRP has already been demonstrated in some preliminary studies [5]. Recently, some authors have described the high sensitivity of DWI in detecting recurrent PCa after brachytherapy [6] and RRP [7].

In this paper, we present a case of $\mathrm{PCa}$ recurrence in the bladder wall after RRP: we report the main features at endorectal-MRI, including DWI and DCE-MRI, and discuss the role of this multi-parametric imaging modality in evaluating patients with a suspected local recurrence of PCa.

\section{Case report}

A 78-year-old man was referred to our institution for biochemical relapse after RRP for a PCa. The PSA level at the moment of the RRP was $10.3 \mathrm{ng} / \mathrm{mL}$; pathological examination showed a $\mathrm{PCa}$ in both prostatic lobes, with a Gleason score (GS) of 3+3, without extra-prostatic extension (pT2c,N0,Mx). Biochemical recurrence occurred 24 months after RRP, with a slow increase of the PSA level, from $0.97 \mathrm{ng} / \mathrm{mL}$ to $1.7 \mathrm{ng} / \mathrm{mL}$ over five years. Based on these clinical data, local recurrence was suspected and endorectal-MRI was performed for investigating the prostatic fossa. This exam was carried out using a $1.5 \mathrm{~T}$ whole-body scanner with a pelvic phased-array surface coil (Signa HDxt; General Electric Company, Milwaukee, WI, USA) combined with a disposable endorectal coil (MedRad, Indianola, Pa), after a cleansing rectal enema. According to our routine protocol, a morphological study was obtained using Fast Relaxation Fast Spin-Echo (FRFSE) T2weighted sequences in the sagittal, transverse and coronal planes, and a Fast Spin-Echo (FSE) T1-weighted sequence in the transverse plane, all including the bladder and the symphysis, with a slice thickness of $4 \mathrm{~mm}$.

The DWI acquisition was performed in the axial plane, using a single-shot echo-planar imaging (SSEPI) sequence, with two b-values $\left(0\right.$ and $\left.600 \mathrm{~s} / \mathrm{mm}^{2}\right)$ and a slice thickness of 4 $\mathrm{mm}$.

The DCE-MRI was obtained using a threedimensional Spoiled Gradient Recalled (SPGR) T1-weighted sequence during the intravenous injection of a contrast bolus of $0.2 \mathrm{ml} / \mathrm{kg}$ of body weight of Gadoterate meglumine (Dotarem, Guerbet S.A., Roissy, France), at a flow rate of 3-4 ml/sec followed by $15 \mathrm{ml}$ of saline solution. Twenty threedimensional data sets, two before and eighteen after contrast administration, were acquired with a 19-second temporal resolution and a total duration of 4-5 minutes. The DWI and DCE-MRI images were processed on an independent workstation with dedicated software (Functool, 4.5.5, General Electric Company). Regions of interest (ROIs) positioned on the suspected areas were used to calculate the corresponding value of the apparent diffusion coefficient (ADC) for DWI, and Time-Intensity (T-I) curves for DCE-MRI.

The accurate analysis of the images showed a small nodule, approximately one centimeter in diameter, within the posterior bladder wall, delineated by the mucosa. This nodule appeared hyperintense on T2- weighted images (Fig. 1A and B) and on $\mathrm{b}=600 \mathrm{~s} / \mathrm{mm}^{2}$ images of the DWI (Fig. 1C). The DCE-MRI showed a hypervascular nodule with a T-I 


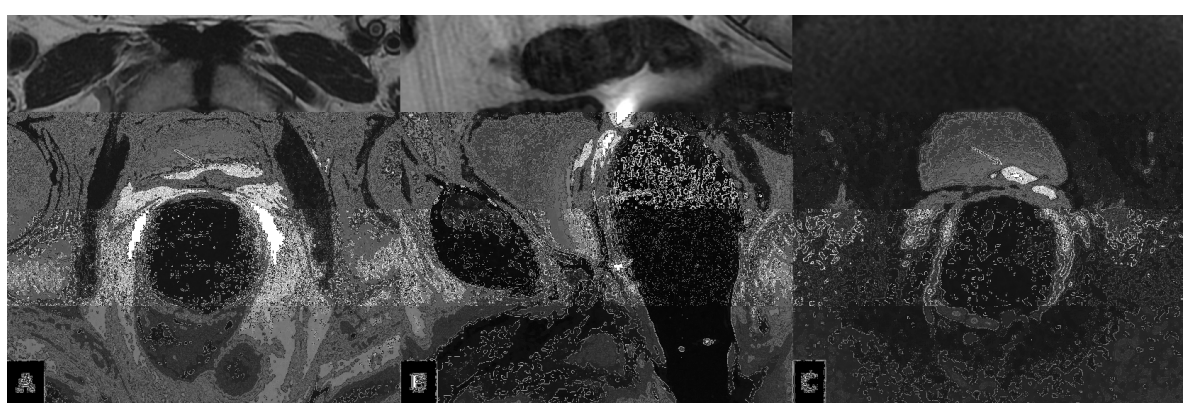

Figure 1: The T2-weighted sequence in the transverse (A) and sagittal (B) planes shows a hyperintense nodule in the posterior wall of the bladder (white arrow). The sagittal view clearly demonstrates that the nodule is distant from the prostatectomy bed (arrowhead in $B$ ). (C) The $b=600 \mathrm{~s} / \mathrm{mm}^{2}$ image of the DWI sequence shows a distinctly hyperintense nodule (white arrow); the corresponding ADC value, calculated on the ADC map (not shown), is reduced $\left(1.48 \times 10^{-9} \mathrm{~m}^{2} / \mathrm{s}\right)$

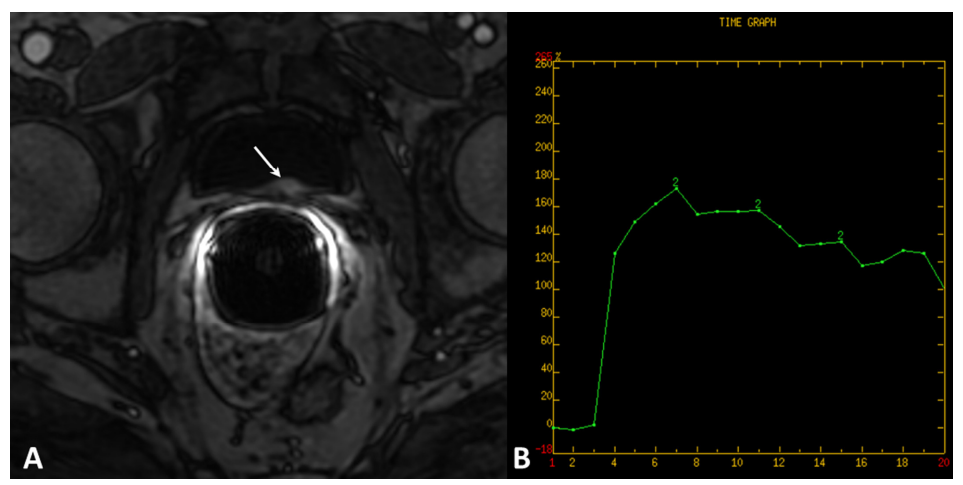

Figure 2: (A) At DCE-MRI, the nodule has intense contrast enhancement after contrast medium administration (white arrow). (B) The corresponding T-I curve is characterized by a prominent rapid wash-in and a rapid wash-out

Based on these imaging findings, PCa recurrence in the bladder wall was suspected. The cystoscopy confirmed the presence of a submucosal nodule in the posterior bladder wall and a radical resection was carried out. At histology, a diagnosis of fibromuscolar tissue infiltrated by PCa (GS $3+3$ ) was made.

\section{Discussion}

Although this is a single case report, we believe that this study points out the ability of multi-parametric MRI to evaluate a local recurrence of PCa after RRP, which affected $20-50 \%$ of patients during a 10 -year followup [1]. In this clinical setting, PSA remains the main tumor marker; the current consensus considers a PSA level $>0.2 \mathrm{ng} / \mathrm{mL}$ indicative of residual/recurrent disease [1]. However, PSA alone is not able to differentiate local from distant recurrence, even if specific features of its increase can reliably accomplish this differentiation; a late biochemical recurrence $(>24$ months after local treatment), low PSA velocity (change of 
PSA over time) and a long PSA doubling time (>6 months) more likely indicate local recurrence; conversely, a rapid biochemical recurrence, high PSA velocity and a short PSA doubling time, more likely indicate distant recurrence $[1,2]$.

In our case, the patient's clinical presentation strongly suggested the presence of local recurrence. In this context, digital rectal examination (DRE) and TRUS frequently demonstrate negative results, even if color/power-Doppler or contrast agents are used [8]. Computed tomography is useful in detecting systemic metastases, but its value in the evaluation of the prostatic fossa is very limited [9].

Endorectal-MRI has been used to detect local recurrence following RRP. While most local recurrences are perianastomotic or retrovesical, 30\% may be found elsewhere in the pelvis, at sites which can be more readily assessed by MRI than by TRUS [1]. Previous studies on the use of T2-weighted MRI alone, reported variable values of sensitivity and specificity $\quad(48-95 \%$ and $52-100 \%$, respectively) [1]; endorectal-MRI also allows clear evaluation of retained seminal vesicle remnants which are present in up to $20 \%$ of patients and can be the site of cancer recurrence.

Recent studies have suggested that newer MR techniques, including spectroscopy and DCE-MRI, can improve the detection of local recurrence [5]. In particular, DCE-MRI seems to be a very promising technique in detecting recurrent cancer foci. The authors of these studies found that DCE-MRI, either alone or in combination with T2-weighted MRI, significantly improved sensitivity and specificity to $84.1-88 \%$ and $89.3-100 \%$ respectively. In addition, Boonsirikamchai et al. [10] attempted to characterize cancer tissue by using T-I curves obtained starting from postprocessed DCE-MRI images.

DW-MRI has recently been introduced as an innovative diagnostic tool in the study of microstructural and functional changes in several pathologies of various organs without contrast medium [6]. In the field of urologic oncology, DW-MRI has various applications, mainly related to the characterization of focal renal masses and the detection of prostate and bladder cancer [7].

Nowadays, the Choline PET/CT may visualize the site of recurrence by revealing both local and systemic recurrence in a single step. In most of the studies, the detection rate is about $30-40 \%$ for disease relapse after radical treatment, even in the presence of low $(<5 \mathrm{ng} / \mathrm{ml})$ PSA [11]. Unfortunately, the main limitation of the Choline PET/CT is the low resolution of scanners that is about 5 $\mathrm{mm}$ [11]; moreover, the detection rate depends on the PSA level and it should be waited in presence of a PSA level of about $1.5-2 \mathrm{ng} / \mathrm{ml}$.

In our case, DW-MRI and DCE-MRI permitted correct evaluation of the nodular tissue in the bladder wall as a PCa recurrence allowing a radical surgical trans-urethral treatment. Moreover, our experience demonstrated that the combination of a T2-weighted sequence with DCE-MRI and DWI is able to detect a local recurrence of $\mathrm{PCa}$, even in the presence of low levels of PSA $(<2 \mathrm{ng} / \mathrm{mL})$.

In conclusion, the endorectal MRI with multiparametric approach (essentially with DCE) may have a role in the early phase of PSA relapse.

\section{References}

1. Katz S, Rosen M (2006) "MR imaging and MR spetroscopy in prostate cancer management," Radiol Clin North A, 44(5):723-734.

2. Schiavina R, Borghesi M, Fiorentino $M$, Brunocilla E, Manferrari F, Vagnoni V, et al, (2013) "Identification of prostate cancer risk categories according to surgical margins status, pathological stage and Gleason score," Int J Urol 20(11):1097-1103. 
3. Leonardo C, Simone G, Papalia R, Franco G, Guaglianone S, Gallucci M, (2009) "Salvage radical prostatectomy for recurrent prostate cancer after radiation therapy," Int J Urol 16(6):584-586.

4. Chalasani V, Iansavichene AE, Lock $\mathrm{M}$, Izawa JI, (2009) "Salvage radiotherapy following radical prostatectomy," Int J Urol 16(1):31-36.

5. Sciarra A, Panebianco V, Salciccia S, Osimani M, Lisi D, Ciccariello M, et al, (2008) "Role of Dynamic Contrast-Enhanced Magnetic Resonance (MR) Imaging and Proton MR Spectroscopic Imaging in the detection of local recurrence after radical prostatectomy for prostate cancer," Eur Urol 54:589-600.

6. Tamada T, Sone T, Jo Y, Hiratsuka J, Higaki A, Higashi H, et al, (2011) "Locally recurrent Prostate Cancer After High-Dose-Rate Brachytherapy: The value of DiffusionWeighted Imaging, Dynamic ContrastEnhanced MRI, and T2-Weighted Imaging in localizing tumors," AJR Am J Roentgenol 197:408-414.

7. Giannarini G, Nguyen DP, Thalmann GN, Thoeny HC, (2012) "Diffusion-weighted magnetic resonance imaging detects local recurrence after radical prostatectomy: initial experience," Eur Urol 61(3):616-620.

8. Drudi FM, Giovagnorio F, Carbone A, Ricci P, Petta S, Cantisani V, et al, (2006) "Transrectal color doppler contrast sonography in the diagnosis of local recurrence after radical prostatectomy comparison with MRI," Ultraschall in Med 27:146-151.

9. Older RA, Lippert MC, Gay SB, Omary RA, Hillman BJ, (1995) "Computed tomography appearance of the prostatic fossa following radical prostatectomy," Acad Radiol 2(6):470-474.

10.Boonsirikamchai P, Kaur H, Kuban DA, Jackson E, Hou P, Choi H, (2012) "Use of maximun slope images generated from dynamic contrast-enhanced MRI to detect locally recurrent prostate carcinoma after prostatectomy: a practical approach," $\mathrm{AJR} \mathrm{Am}$ J Roentgenol 198(3):W228-236.

11.Schiavina R, Ceci F, Borghesi M, Brunocilla E, Vagnoni V, Gacci M, Castellucci P, Nanni C, Martorana G, Fanti S, (2013) "The dilemma of localizing disease relapse after radical treatment for prostate cancer: which is the value of the actual imaging techniques?," Curr Radiopharm 6;6(2):92-95. 